\title{
THE PREVENTION AND TREATMENT OF ANAPLASMOSIS
}

\author{
By James G. Miller \\ Depariment of Pharmacology, University of Michigan, Ann Arbor, Mich.
}

\section{Introduction}

There have been at least 80 drugs proposed for the treatment of anaplasmosis. The suggested efficacy of these agents has often been based on a small number of clinical cases, with an almost complete lack of prior information as to their specific activity, or lack of such activity, against Anaplasma marginale. The host specificity of the parasite has defeated many efforts to evolve a screening method that would adapt itself to the examination of a large number of compounds. The previous lack of a preliminary screen has been a major stumbling block in the rational chemotherapeutic approach to the problem.

\section{Methods of Study}

It is difficult to obtain clinical cases of anaplasmosis in ruminants other than the adult bovine, and attempts to grow even Anaplasma marginale in other hosts or in artificial media have not been successful. The role of the spleen in the host's response to anaplasmosis has long been recognized. An adult resistant carrier animal will undergo a relapse following splenectomy; and calves, which at best exhibit only mild symptoms of the disease, become susceptible to acute anaplasmosis when splenectomized. This has lead to the use of splenectomized calves for the chemotherapeutic screening procedure.

In the following experiments, male calves were splenectomized between 10 and 14 days of age, and then inoculated with Anaplasma marginale 2 weeks later. The inoculum consisted of a subcutaneous injection of $5 \mathrm{cc}$. of citrated whole blood. A common carrier source was used throughout these experiments. This carrier was determined to have the most virulent strain of $A n a-$ plasma marginale available and, in fact, one of the most virulent that had ever been encountered.

Various phases of the disease in splenectomized calves were examined by Lotze ${ }^{2}$ and also at Louisiana State University, ${ }^{3}$ Baton Rouge, Louisiana, to determine their adaptability to a screening method. The incubation period varied widely even when, as in these experiments, a constant volume of inoculum from a single source was used.

It should be pointed out that the incubation period is usually not longer than 60 days and that it can be entirely abolished by massive transfusions of highly infected blood. This wide variation in the incubation period makes it an undesirable criterion of drug activity. Similarly, the peak percentage of infested red blood cells cannot be utilized, as it varies widely from animal to animal. A peak infestation of 30 per cent of the red blood cells is likely to result in just as severe symptoms and anemia as in the instance of a red cell infestation reaching 85 per cent. A splenectomized calf that survives the initial attack of anaplasmosis often undergoes a recurrent series of relapses of varying degrees of severity, so that this portion of the disease is not adaptable to the screening 
TABLE 1

Variations in the Incubation Period of 68 Splenectomized Calves*

\begin{tabular}{c|c|c|c}
\hline \hline Incubation period & No. of animals & Incubation period & No. of animals \\
\hline 11 days & 1 & 25 days & 3 \\
12 days & 3 & 26 days & 2 \\
14 days & 6 & 27 days & 1 \\
15 days & 4 & 28 days & 2 \\
16 days & 2 & 29 days & 2 \\
17 days & 3 & 33 days & 1 \\
18 days & 3 & 34 days & 1 \\
19 days & 2 & 39 days & 1 \\
20 days & 7 & 40 days & 1 \\
21 days & 2 & 41 days & 2 \\
22 days & 6 & 44 days & 1 \\
23 days & 4 & & \\
\hline \hline
\end{tabular}

* Range: 11 to 44 days. Mean: 22 days. Standard deviation: \pm 7 days.

program. The only portion of the disease in splenectomized calves that is consistent enough to be used as a criterion for specific drug activity is the rate of infestation of red blood cells. The percentage of infested red blood cells doubles approximately every 24 hours until a peak is reached (FIGURE 1). For a compound to exhibit specific activity against the Anaplasma marginale, it would have to alter this pattern of cell infestation.

\section{Results}

The drugs previously used in the therapy of this disease can be classified as arsenicals, antimalarials, antimony derivatives, and dyes. Two antimalarials, chloroquine dihydrochloride ${ }^{4}$ and paludrine diphosphate, ${ }^{5}$ were subjected to screening with splenectomized calves. In both instances, very high dosage levels failed to alter the course of red blood cell infestation (FIGURE 2) and were adjudged to be lacking specific activity against $A$ naplasma marginale.

Chlortetracycline and oxytetracycline were also screened. Chlortetracycline was administered intravenously at the rate of $5 \mathrm{mg}$./ lb. body weight at various stages of erythrocyte infestation. In all instances there was no further increase in the level of infestation following treatment (FIGURE 3). Oxytetracycline gave similar results when administered intravenously at $2.5 \mathrm{mg} . / \mathrm{lb}$. body weight. There was 100 per cent recovery in 32 animals treated with these antibiotics, as contrasted with 80 per cent fatalities in 24 untreated splenectomized calves. ${ }^{3}$ Chloramphenicol therapy resulted in a temporary inhibition of red blood cell infestation, which was confined to the period of treatment. Subsequent cell infestation often reached 80 to 90 per cent of all erythrocytes. ${ }^{6}$

From these experiments, it was apparent that chlortetracycline and oxytetracycline had a demonstrable effect on Anaplasma marginale. Further experiments were conducted to outline the parameters of this action, and its possible utility, if any, in the treatment of this disease. Subinoculations from chlortetracycline- and oxytetracycline-treated calves indicated that they were still carriers of virulent Anaplasma marginale. ${ }^{3}$ Several attempts were made to 


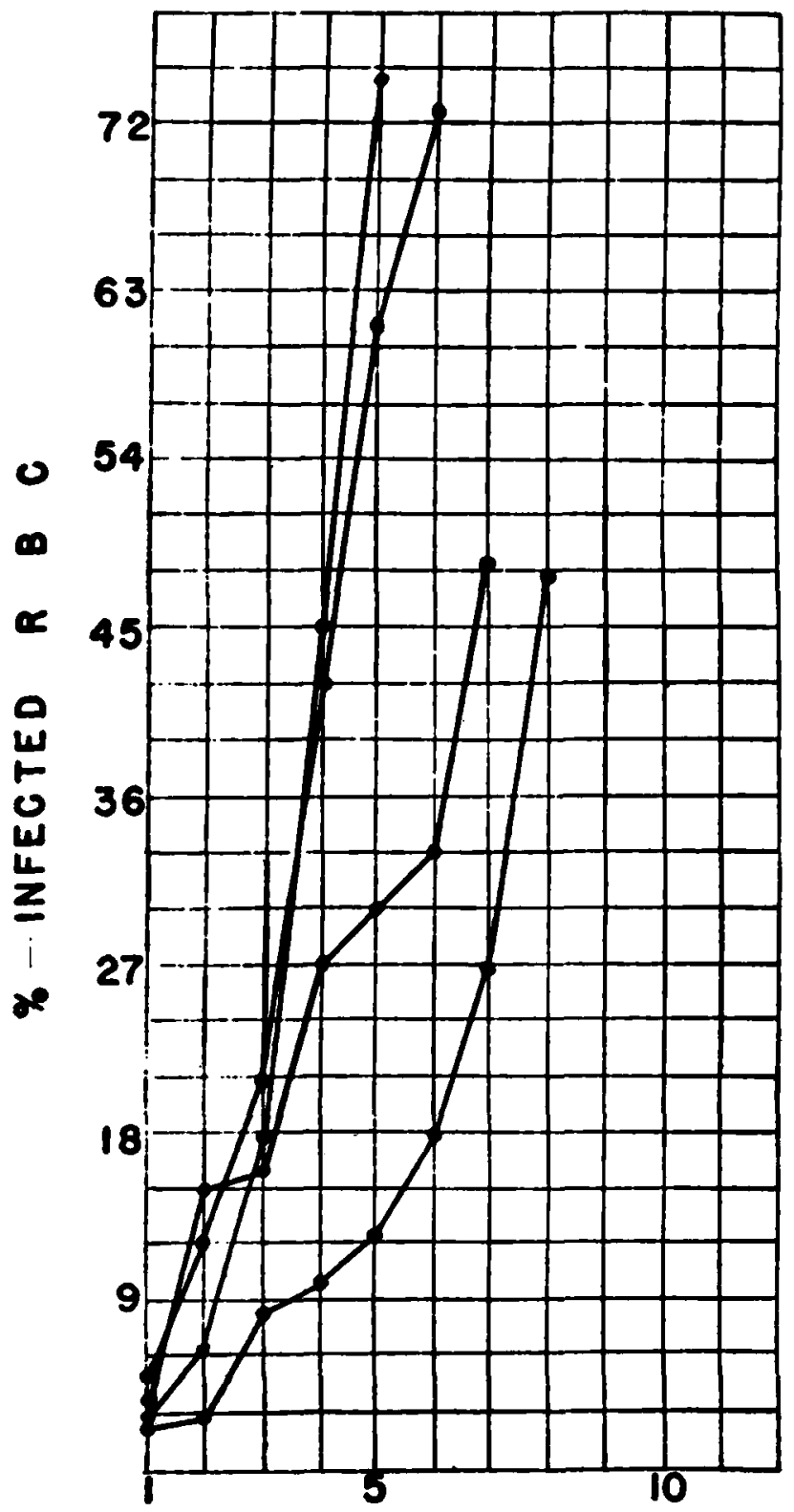

DAYS FROM I\% INFECTION

FIGURE 1. A graph to show the rapid increase of the percentage of infected red blood cells in four untreated splenectomized calves inoculated with $5 \mathrm{cc}$. of whole blood from the common carrier. The activity of chemotherapeutic agents against the Anaplasma marginale is evaluated by their ability to halt or modify this trend (from Miller et al.3). 


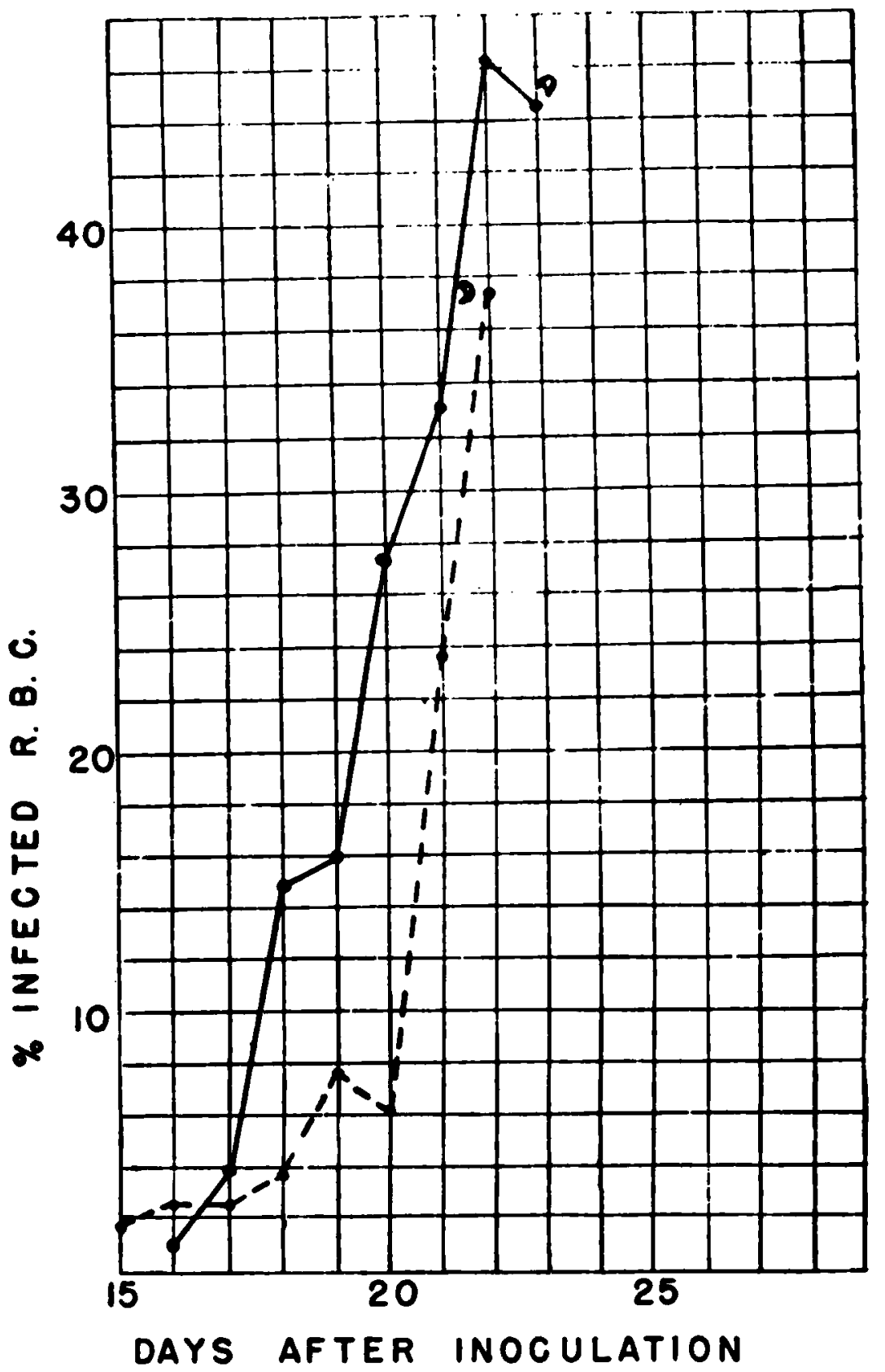

\section{untreated control; - - - treuted calf.}

Figure 2. The results of continuous treatment with chloroquine dihydrochloride during the patent phase of anaplasmosis in a splenectomized calf. The dosage was $5.8 \mathrm{mg}$./lb. daily, administered intravenously in a divided dose four hours apart (from Miller et al.4). 


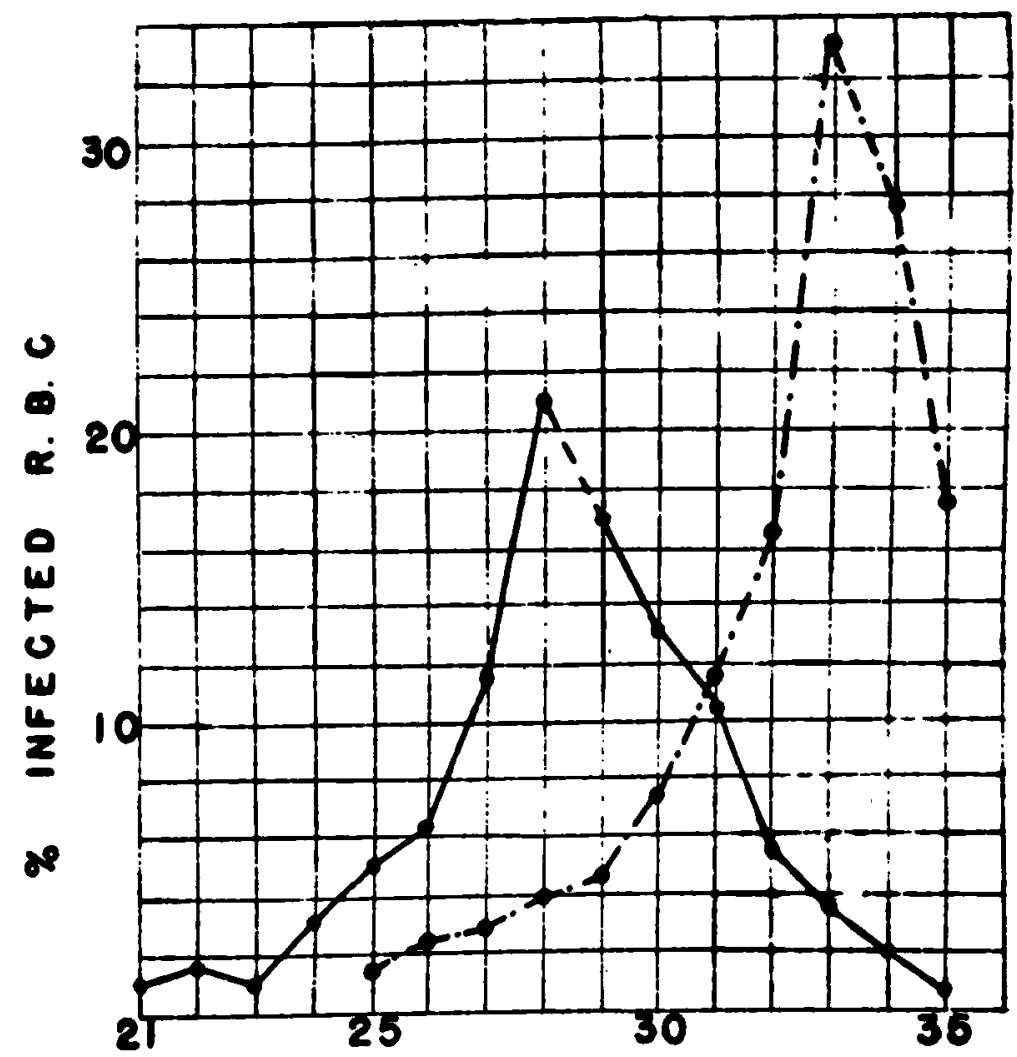

DAYS AFTER INOCULATION

FIGURE 3. A graph to show the result of a single dose of chlortetracycline on the increase of infected red cells. The solid line indicates the percentage of infected red cells in the treated calf. The broken portion of that line indicates the day on which chlortetracycline was given. The dotted line indicates the percentage of infected red cells of the untreated control calf (from Miller et al.3).

sterilize carriers of anaplasmosis using large doses of chlortetracycline over a short period of time. These experiments failed to rid carriers of the infection, ${ }^{7}$ in spite of the fact that the blood of these animals was free of infective Anaplasma marginale for a brief period following therapy. ${ }^{8}$ Similar results were obtained when chlortetracycline and oxytetracycline were administered at the rates of 5 and $2.5 \mathrm{mg}$./lb. body weight, intravenously, for periods of 16 and 20 days respectively. ${ }^{3}$ Subsequent experiments with both antibiotics, at increased dosage levels over a period of 20 days,${ }^{10}$ resulted in sterilization of the blood of carrier animals for at least 100 days and up to 360 days, at the last determination. This was accompanied by the disappearance of Anaplasma marginale complement-fixing antibodies at an early date following therapy.

The possible prophylactic application of these antibiotics was explored. Certainly, if pretreatment could prevent or ameliorate an epizootic, a very real advance would have been made. It soon became apparent, however, that admin- 
istration of chlortetracycline early in the prepatent phase of the disease served only to delay the onset of clinical symptoms $s^{9}$ far beyond the usual incubation period, without any advantageous effect on the acute phase. Treatment with chlortetracycline and oxytetracycline early in the patent phase of the disease, that is, between 1 and 10 per cent of infested erythrocytes, resulted in no further increase in red blood cell infestation, amelioration of clinical symptoms, and no subsequent relapses up to 120 days following therapy. ${ }^{3}$ Animals treated in this manner, however, of ten developed an undesirable degree of anemia.

In 1952, a survey of clinical cases of anaplasmosis was conducted in Louisiana." One hundred and thirty-two cases of the disease were available for study. Blood samples were collected at the time of treatment, and red and white blood cell counts, red blood cell volume, hemoglobin content, and degree of erythrocyte infestation were determined. Sixty of these animals were treated with chlortetracycline and 52 with oxytetracycline. Twenty animals received the treatment that was currently employed by the attending veterinarian. These treatments were classified as 'nonspecific,' as they were supportive in nature; medication involved calcium gluconate, sodium caccodylate, and strychnine sulfate.

\section{Discussion}

An initial screening procedure is fundamental in the chemotherapeutic approach to anaplasmosis. Although the multiplicity of suggested treatments is regrettable, it is understandable in view of the lack of a satisfactory preliminary screen for specific activity. The use of the splenectomized calf makes available an experimental tool for the study of anaplasmacidal or Anaplasma inhibitory activity, but this is not an entirely satisfactory approach. The chief disadvantages of this method are the expense and time involved. It is therefore entirely unsuited to the mass screening approach, where hundreds of chemical structures are to be tested, but it is applicable to careful and considered experimentation with a few drugs. In the author's opinion, there is little doubt that the results obtained by this method are valid. In no instance has an apparent specific activity of a drug in the splenectomized calf failed to be substantiated in the adult nonsplenectomized animal, nor has the lack of activity in the experimental animal been confronted by activity in the adult.

The specific activity of a drug against Anaplasma marginale in the splenectomized calf does not mean that the particular agent will be of value in the therapy of the disease. This in itself is a problem that must be answered in the clinical environment. The interpretations of such clinical studies, if properly planned, will be greatly aided by the information obtained from the screening program. Certainly the mere recovery of a certain percentage of a group of treated animals is an inadequate criterion in the therapy of many diseases, including anaplasmosis.

One of the most striking features observed in the clinical trials in Louisiana was the late stage of the disease at which the animals were presented for treatment. Often the animal was afebrile, jaundiced, anemic, with a low level of erythrocytes infected. Seventy-two per cent of all animals in the field trial had less than $4 \mathrm{gm}$. of hemoglobin per $100 \mathrm{cc}$. of blood; this may be compared 
with a similar survey in Oklahoma ${ }^{12}$ in which only 14 per cent of the animals had less than this amount of hemoglobin. These differences, explainable perhaps on the basis of a less virulent form of anaplasmosis, or because of the epizootic rather than the sporadic proportions of the disease in Oklahoma, serve to illustrate the varying conditions under which anaplasmosis is treated. It is doubtful that anaplasmosis can be effectively treated in the postfebrile, acutely anemic period with a specific chemotherapeutic agent. The rapid red cell infestation, which could have been halted by chlortetracycline or oxytetracycline, has already taken place. The phase of the disease that should be given immediate attention is the acute anemia. Treatment may logically embody repeated transfusions of whole blood, hematopoietic stimulating therapy, and nursing care.

At this time, protection of susceptible animals against anaplasmosis is not a practical procedure in this country. Young animals inoculated with Anaplasma marginale become resistant to further infection, but remain carriers of the disease. This carrier status discourages the widespread inoculation of young animals, particularly in view of the susceptible cattle population in many areas.

Chlortetracycline and oxytetracycline have been used prophylactically to avert the crippling economic losses associated with an epizootic of anaplasmosis. This prophylactic use is based on the treatment of animals in the early patent phase of the disease, when Anaplasma marginale are present in less than 10 per cent of the erythrocytes. The examination of blood smears every five days proved to be a suitable basis for the initiation of therapy. This approach is naturally limited to those instances where an epizootic is imminent in a small group of easily handled susceptible animals.

\section{References}

1. Piercy, P. L. 1950. Personal communication.

2. LOTZE, J. C. 1947. Variables and constants in experimental bovine anaplasmosis and their relationship to chemotherapy. Am. J. Vet. Research 8(28): 267-274.

3. Milier, J. G., H. E. Levy, B. J. Torbert \& W. T. Oglesby. 1952. A method of screening drugs to be used in the treatment of anaplasmosis-results of testing with Aureomycin and Terramycin. Proc. Am. Vet. Med. Assoc. 89th. Ann. Meet. : 160-167.

4. Miller, J. G., H. E. Levy \& W. T. Oglesby, 1953. A report of the screening of aralen dihydrochloride for activity against Anaplasma marginale. Am. J. Vet. Research. 14 (53): 517-519.

5. SplitTER, E. J. 1949. Anaplasmosis treatment with paludrine. J. Am. Vet. Med. Assoc. 114: 224-225.

6. MilleR, J. G. 1952 . Unpublished data,

7. Pearson, C. C. \& W. E. Brock. 1953. Further studies on the use of Aureomycin in anaplasmosis carrier infection. North Am. Veterinarian. 34(6): 408-412.

8. Foote, L. E. \& M. WUL. 1952. Anaplasmosis carrier infection destroyed with Aureomycin. North Am. Veterinarian. 33(6): 406-408.

9. Foote, L. E., H. Farley \& B. Gallagher. 1951. The use of Aureomycin in anaplasmosis. North Am. Veterinarian. 32(8): 547-549.

10. Splitter, E. J. \& J. G. Miller. 1953. The apparent eradication of the anaplasmosis carrier state with antibiotics. Vet. Med. 48(12): 486-488.

11. Miller, J. G., H. E. Levy, B. J. Torbert \& W. T. OgLesby. 1953. The treatment of anaplasmosis in Louisiana with Aureomycin and Terramycin. J. Am. Vet. Med. Assoc. 122(914): 390-392.

12. SMith, H. C., \& D. E. Howell. 1945. Hemoglobin tests on 175 cases of anaplasmosis. Vet. Med. 40: 272-273. 